\title{
Relação entre a biometria ultrassonográfica ocular e os parâmetros morfométricos do crânio, idade, peso e gênero em gatos domésticos ${ }^{1}$
}

\begin{abstract}
Marco A. Ferreira² ${ }^{2}$ Norma Allemann ${ }^{3}$, Luís G.G.G. Dias ${ }^{4}$ e Cristiane dos Santos Honsho ${ }^{4 *}$
ABSTRACT.- Ferreira M.A., Allemann N., Dias L.G.G.G. \& Honsho C.S. 2014. [Relation between opfthalmic ultrasound biometry and the morphometric parameters of the skull, age, weight and gender in domestic cats.] Relação entre a biometria ultrassonográfica ocular e os parâmetros morfométricos do crânio, idade, peso e gênero em gatos domésticos. Pesquisa Veterinária Brasileira 34(2):192-198. Mestrado em Medicina Veterinária de Pequenos Animais, Universidade de Franca, Av. Dr. Armando Salles Oliveira 201, Pq. Universitário, Franca, SP 14404-600, Brazil. E-mail: crishonsho@unifran.br

Ocular ultrasonography is a helpful ophthalmic examination, performed on the absence of transparency in ocular means and frequently requested prior intraocular surgery. Unfortunately, the lack of studies upon ultrasonografic images of the normal feline eye, difficults the evaluation of its biometry and its inner structures in many ophthalmic diseases and also before surgery. The objective of this study was to evaluate the ocular biometry and the morphometric parameters of the cranium of 40 healthy adult cats, including 22 Persian cats (Brachycephalic group - BG) and 18 Mixed-breed cats (Noun brachycephalic group - NBG). $\mathrm{B} / \mathrm{A}$-mode ultrasonographic biometry, utilizing a $9 \mathrm{MHz}$ probe, was performed for depth of the anterior chamber (D1), lens axial length (D2), depth of the vitreous chamber (D3) and axial length of the globe (D4). Morphometric parameters of the cranium were obtained for bizygomatic (BZ) and occipital frontal (OF) diameters. Data was collected and statistical analysis, including " $t$ " paired tests, variance and covariance analysis and multiple linear regressions was performed for every obtained measure and also for animal ages, weigh and gender. Statistic relevant values were observed in D4 parameter for female cats of BG. Multiple linear regression indicated some influence of the weigh, age and OF in D1, D2 and D4 of PG; and of BZ in D1, D3 and D4 of NBG. Besides that, it was observed that biometry was also influenced by weigh, age and morphometric parameters of their craniums in both groups.
\end{abstract}

INDEX TERMS: Skull, brachycephalic, feline, ocular biometry, ultrasonography.

RESUMO.- 0 exame ultrassonográfico ocular é indispensável no pré-operatório de procedimentos cirúrgicos intraoculares como a facectomia, além de ser uma ferramenta

\footnotetext{
${ }^{1}$ Recebido em 21 de outubro de 2013.

Aceito para publicação em 23 de fevereiro de 2014.

${ }^{2}$ Docente da Faculdade de Ciências Sociais e Agrárias de Itapeva (FAIT), Rua João Basso 157, Apto 54, São Bernardo do Campo, SP 09721-100, Brasil. E-mail: kaienavetmarco@gmail.com

${ }^{3}$ Docente do Departamento de Oftalmologia, Escola Paulista de Medicina, Universidade Federal de São Paulo (Unifesp/EPM), Rua Botucatu 821, São Paulo, SP 04023-062, Brasil. E-mail: norma.allemann@pobox.com

${ }^{4}$ Docente do Mestrado em Medicina Veterinária de Pequenos Animais, Universidade de Franca (Unifran), Rua Dr. Armando Salles de Oliveira 201, Franca, SP 14404-600, Brasil. *Autor para correspondência: crishonsho@unifran.br
}

complementar ao exame oftalmológico, em casos de perda da transparência dos meios ópticos. A inexistência de estudos acerca de padrões de normalidades para as medidas do bulbo ocular e de suas estruturas internas nos gatos, cujos valores possibilitam o monitoramento de enfermidades e auxiliam em procedimentos cirúrgicos motivaram este estudo. Utilizaram-se 40 gatos, adultos, machos e fêmeas, livres de enfermidades sistêmica e oftalmológica. Destes, 22 eram da raça persa (grupo braquicefálico - GB) e 18 sem raça definida (grupo não braquicefálico - GNB). A biometria ultrassonográfica ocular transcorneana foi realizada, em modo-B/A, com o transdutor microlinear de $9 \mathrm{MHz}$ e as medidas D1 (profundidade da câmara anterior), D2 (diâmetro do cristalino), D3 (profundidade da câmara vítrea) e D4 (diâmetro axial do bulbo ocular) aferidas. Ainda, men- 
suraram-se as distâncias fronto-occipital e bizigomática e o peso desses animais. Os dados obtidos foram analisados pelo teste- $t$ pareado, seguindo-se as análises de variância e covariância, além da regressão linear múltipla relacionando-se as medidas de D1, D2, D3 e D4 às medidas bizigomática e fronto-occipital, como também à idade, ao peso e ao gênero. Obteve-se como resultado a média de D1, D2, D3 e D4, assim como dos diâmetros bizigomático e fronto-occipital, idade e peso, verificando-se diferenças significativas para D4 nas fêmeas de GB. Houve, pela análise de regressão linear, influência do peso, idade e diâmetro fronto-occipital sobre D1, D2 e D4 nos gatos do GB, e dos diâmetros bizigomático sobre D1, D3 e D4 nos gatos do GNB. Conclui-se que houve diferença no diâmetro axial do bulbo ocular nas fêmeas do GB, e que o peso, a idade e os diâmetros cranianos influenciam a biometria ocular dos gatos braquicefálicos e não braquicefálicos.

TERMOS DE INDEXAÇÃO: Crânio, braquicefálico, ecobiometria, felino, ultrassonografia.

\section{INTRODUÇÃO}

A ultrassonografia ocular na medicina é considerada ferramenta importante para avaliação de casos de má formações assim como de cálculos de lente intraocular, com tanto, permite-se com a mesma técnica em medicina veterinária, complementar o exame semiológico oftalmológico, mormente em casos de perda da transparência dos meios ópticos ou nos casos em que a avaliação do conteúdo intraocular (Soares et al. 1998, Atta 1999, Corrêa et al. 2002, Hijar 2008) ou intraorbitário (Adan et al. 2001) é necessária. Trata-se de técnica indispensável ao pré-operatório da facectomia (Corrêa et al. 2002, Lupinacci et al. 2004, Williams 2004, Bhatt et al. 2008, Junior 2008, Silva et al. 2010) pois, pela avaliação da lente, obtêm-se valores de sua topografia, apresentação morfológica (Williams 2004), sendo possível identificar lesões no polo posterior do bulbo ocular como a persistência da túnica vascular (Tartarella et al. 2013) e descolamentos de retina secundários à catarata (Lucena et al. 2009, Gomes et al. 2013), aspectos estes relevantes à programação e escolha do método cirúrgico (Martins et al. 2010). O ultrassom pode auxiliar na condução de procedimentos de caráter invasivo, como a citologia aspirativa e o bloqueio retrobulbar (Kubal 2008, Luyet et al. 2008). Além disso, constitui técnica de exame não invasivo, acessível e de rápida realização em cães e gatos (Corrêa et al. 2002). Na medicina veterinária, a primeira publicação sobre biometria ultrassonográfica ocular em cães foi realizada por Schiffer et al. (1982). Em 1984, Nathan et al. realizaram a descrição da biometria ocular em felinos sem raça definida em diferentes faixas etárias, incluindo valores de refração óptica. Até o momento, já se encontram disponíveis valores biométricos ultrassonográficos do bulbo do olho de jacarés (Maia et al. 2003), ferrets (Hernandes-Guerra et al. 2007), elefantes (Nunnery et al. 2008), cabras (Ribeiro et al. 2009, Ribeiro et al. 2010), papagaios (Lehmkuhl et al. 2010) e chinchilas (Lima et al. 2010). Recentemente, Baraldi et al. (2012) realizaram a avaliação em modo-A e modo-B das dimensões do bulbo ocular em felinos sem raça definida, verificando diferenças presentes entre os dois métodos de mensuração das medidas oculares.

A curvatura e a espessura corneal apresentam importância ultrassonográfica, em situações relacionadas à refração, sendo que a avaliação da curvatura corneana associada à mensuração do comprimento do cristalino e à retinoscopia são importantes ferramentas para identificar o erro refrativo dos animais (Murphy et al.1992), porém as cirurgias refrativas, que visam à correção perfeita da emetropia em animais, pouco recebem atenção (Miglior et al. 2004). De acordo com a descrição de Mutti et al. (1999) o valor de maior alongamento da câmara vítrea avaliado pela ultrassonografia ocular influencia na ocorrência da miopia em cães da raça labrador.

A inexistência de dados acerca dos valores de referência da biometria ultrassonográfica ocular para os gatos braquicefálicos e daqueles sem raça definida, ou ainda, o estabelecimento de correlação entre as medidas oculares e os parâmetros morfométricos do crânio, a idade, o peso e o gênero motivaram o desenvolvimento deste estudo.

\section{MATERIAL E MÉTODOS}

\section{Aspectos éticos}

A pesquisa foi conduzida obedecendo-se aos critérios da $A s$ sociation for Research in Vision and Ophthalmology (ARVO) (ARVO 2012) e sob a anuência dos proprietários e aprovação do Comitê de Ética no Uso de Animais (CEUA) da Universidade de Franca (protocolo 057/11, aprovado em 15/12/2011).

\section{Animais e grupos experimentais}

Foram utilizados 40 gatos adultos, machos e fêmeas, livres de alterações oftalmológicas. Os animais foram distribuídos segundo a conformação do crânio, em dois grupos: grupo braquicefálico, cujo padrão de crânio consiste em menor distância fronto-occipital e, neste estudo, foi representado por gatos da raça persa (GB, $\mathrm{n}=22$ ), oriundos de gatil comercial; e grupo não braquicefálico, cujo padrão morfológico do crânio não se enquadra no grupo braquicefálico (GNB, $n=18$ ). Compuseram este grupo, gatos sem raça definida originários de atendimento especializado em clínica particular. Constituíram o GB seis machos com idade de 5,50 $\pm 2,42$ anos e pesando $3,53 \pm 0,70 \mathrm{~kg}$, e 16 fêmeas com $6,06 \pm 2,64$ anos de idade e pesando $3,07 \pm 0,48 \mathrm{~kg}$. 0 GNB foi composto por oito machos com idade de $5,62 \pm 4,40$ anos e peso de $4,58 \pm 1,31 \mathrm{~kg} \mathrm{e}$ 10 fêmeas com 7,10 $\pm 3,98$ anos de idade, pesando $3,91 \pm 0,68 \mathrm{~kg}$.

\section{Avaliações}

Ultrassonografia ocular e parâmetros morfométricos. A biometria ultrassonográfica ocular foi realizada pela técnica de contato direto, transcorneano, com auxílio de transdutor microlinear de $9 \mathrm{MHz}$, conectado ao aparelho de ultrassom veterinário (Kaixin Xl $5000^{\circledR}$ Hong-Kong, CHN). As imagens foram obtidas através de corte transversal-horizontal, em modo-A e modo-B, com o transdutor posicionado no centro da córnea. Previamente ao exame foi instilada uma gota de colírio anestésico à base de proximetacaína (Anestalcon ${ }^{\circledR}$, Alcon do Brasil SA. São Paulo/SP, Brasil), promovendo assim a dessensibilização da córnea. Após 10 minutos, a superfície ocular fora recoberta com gel (Gelsound Hidrogel ${ }^{\circledR}$, São Paulo/SP) para ultrassonografia (Fig.1). Para padronização do exame ultrassonográfico ocular, todos os exames foram iniciados pelo bulbo ocular direito e todas as medidas foram realizadas por apenas um examinador.. Os gatos foram contidos manualmente e permaneceram em decúbito esternal duran- 


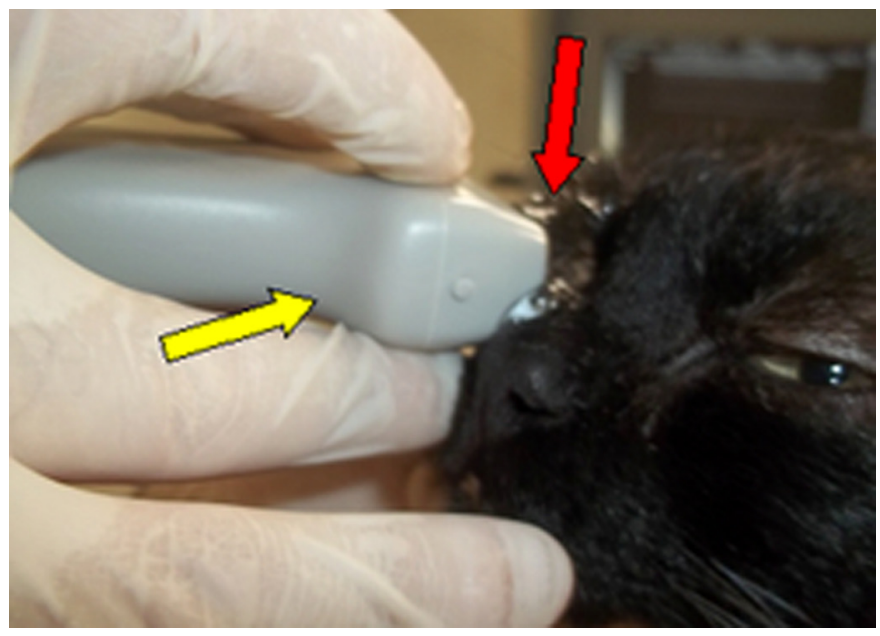

Fig.1. Imagem fotográfica de exame ultrassonográfico ocular em gato sem raça definida. Observa-se transdutor microlinear 9 $\mathrm{MHz}$ (seta amarela) em contato com o olho direito tendo por interface gel para ultrassonografia (seta vermelha).

te o exame. As imagens obtidas foram armazenadas para futura análise.

Os parâmetros avaliados à biometria ocular foram: D1- pro- fundidade da câmara anterior (distância entre a face interna da córnea e a cápsula anterior do cristalino), D2- espessura do cristalino (distância entre cápsula anterior e a cápsula posterior), D3profundidade da câmara vítrea (distância da cápsula posterior do cristalino e a parede posterior do bulbo ocular) e D4- comprimento axial do bulbo ocular (distância entre a face externa da córnea até a parede posterior do bulbo ocular) (Fig.2).

Para obtenção dos diâmetros fronto-occipital (Fig.3A) e bizigomático (Fig.3B), utilizou-se paquímetro (MTK ${ }^{\circledR}$, Mytutoiu, São Paulo/SP), com precisão de $0,05 \mathrm{~mm}$ e nivelador de superfície. 0 registro do peso foi realizado valendo-se de balança digital veterinária (Mt 3000 ${ }^{\circledR}$, Med-Sinal, São Paulo/SP).

Análise estatística. Empregou-se o teste- $t$ pareado para verificar diferenças entre os olhos esquerdo e direito do mesmo animal, em seguida comparam-se as medidas de D1, D2, D3 e D4, entre os grupos (GB e GNB).

Para a comparação entre as medidas oculares de D1, D2, D3 e D4 entre os grupos foi realizada análise de variância (ANOVA). Diferenças entre as medidas oculares em relação ao gênero foram avaliadas separadamente para cada raça, por meio de análise de covariância (ANCOVA), nesta análise, diferenças para cada medida ocular D1, D2, D3 e D4 foram investigadas em relação ao gênero, utilizando como covariáveis as medidas de idade, massa corpórea, diâmetro fronto-occipital e diâmetro bizigomático. Adotou-se nível de significância $\mathrm{P}<0,05$.

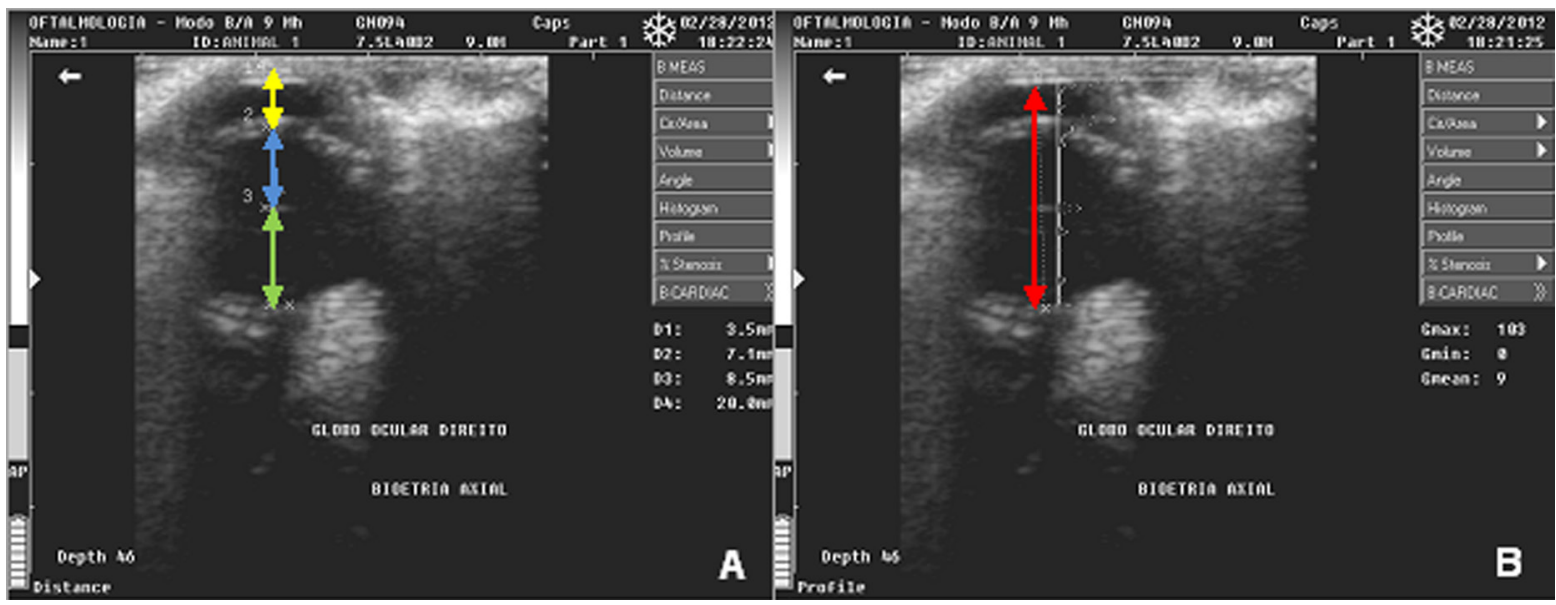

Fig.2. Imagens ultrassonográficas axiais do bulbo ocular de gato da raça persa. Observa-se na Figura A (seta amarela = D1, seta azul = D2, seta verde = D3) e Figura B modo-A (seta vermelha $=$ D4).

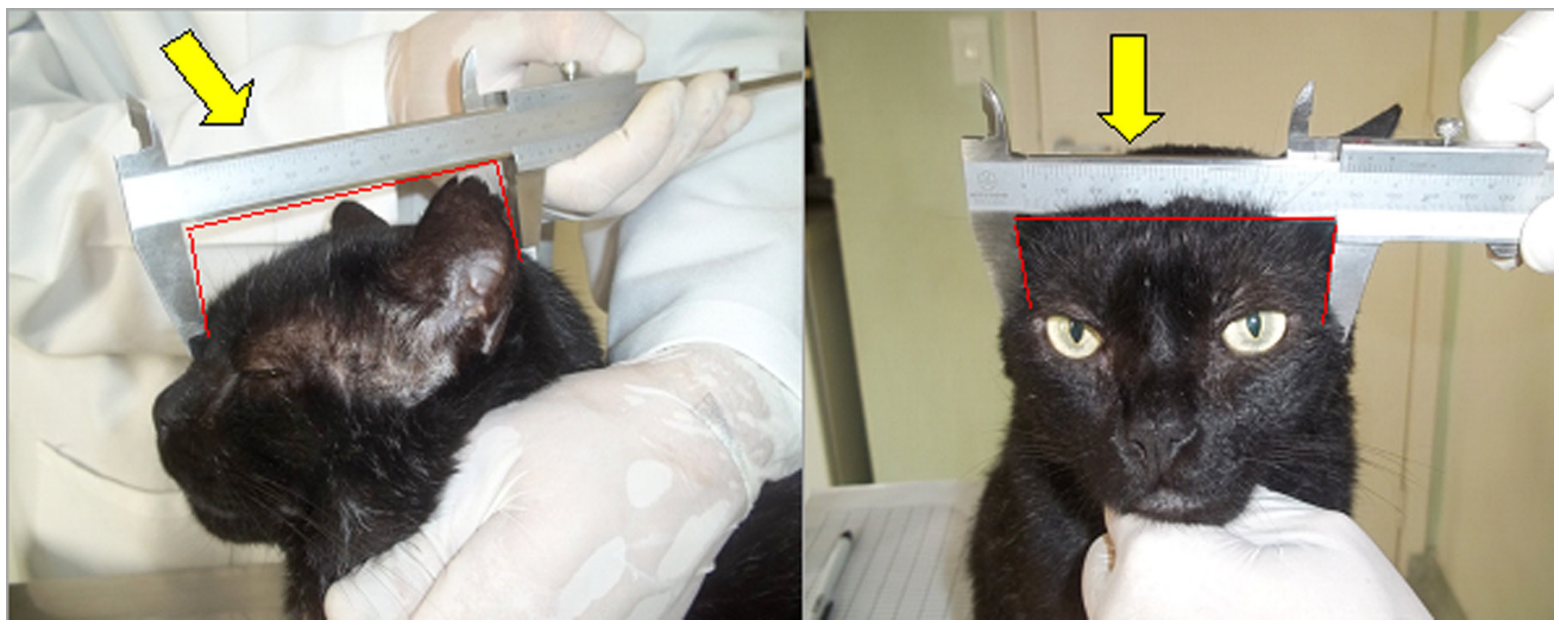

Fig.3. Imagens fotográficas de gato sem raça definida. (A) Mensuração do diâmetro fronto-occipital com paquímetro (seta), vista lateral. (B) Mensuração do diâmetro bizigomático com paquímetro (seta), vista frontal. 
Relações entre as medidas oculares e idade, massa corpórea, diâmetro fronto-occipital e diâmetro bizigomático foram verificadas por meio de análise de regressão linear múltipla. Estas análises foram realizadas separadamente para os grupos GB e GNB, sendo que apenas para a medida ocular de D4 esta análise foi realizada separadamente também para machos e fêmeas. Nesta análise realizou-se o método por etapas (Stepwise), com critérios de entrada e saída de variáveis, respectivamente, valores de $\mathrm{P}$ de 0,05 e 0,10. Em cada análise utilizou-se como variáveis dependentes as medidas oculares e como variáveis preditoras a idade, a massa corpórea, o diâmetro fronto-occipital e o diâmetro bizigomático. As relações significativas foram apresentadas, juntamente com intervalo de confiança para cada parâmetro do modelo.

Para todos os testes realizados o valor de alfa foi de 0,05 , utilizando correção de Bonferroni para múltiplos testes quando necessário.

\section{RESULTADOS}

A análise pelo teste- $t$ pareado para as medidas oculares de $\mathrm{D} 1(\mathrm{P}=0,12)$, $\mathrm{D} 2(\mathrm{P}=0,64), \mathrm{D} 3(\mathrm{P}=0,60)$ e $\mathrm{D} 4(\mathrm{P}=0,41)$ não identificou diferenças entre olhos direito e esquerdo em ambos os grupos.

A comparação das medidas biométricas oculares entre GB e GNB pela análise de variância (ANOVA) estão descritas no Quadro 1.

As comparações das medidas oculares entre os gêneros mostraram diferenças significativas apenas para o diâmetro axial do bulbo ocular D4 em GB $(\mathrm{P}<0,001)$, porém nas demais medidas D1, D2 e D3 não demonstraram diferenças com relação ao gênero (Quadro 2).

Para GB, as medidas oculares referentes à câmara anterior (D1) do bulbo do olho, puderam ser previstas significativamente por uma combinação do peso e idade dos animais (Quadro 3). A espessura do cristalino (D2) apresentou relação significativa com a idade (Quadro 3) e o diâmetro axial do bulbo ocular (D4) com o peso (kg) das fêmeas e com o diâmetro bizigomático em machos (Quadro 3). As

Quadro 1. Médias \pm desvios-padrão para as medidas de todos os olhos, em milímetros, da câmara anterior (D1), tamanho do cristalino (D2), profundidade da câmara vítrea (D3) e diâmetro axial do olho (D4), em GB e GNB $(P<0,05)$

\begin{tabular}{cccc}
\hline & GB & GNB & P \\
\hline D1 & $3,08 \pm 0,54$ & $3,65 \pm 0,43$ & $<0,001^{*}$ \\
D2 & $8,01 \pm 0,55$ & $7,63 \pm 0,60$ & $0,003^{*}$ \\
D3 & $7,92 \pm 0,62$ & $7,78 \pm 0,60$ & 0,274 \\
D4 & $19,31 \pm 0,57$ & $19,22 \pm 0,84$ & 0,546
\end{tabular}

Quadro 3. Parâmetros, coeficiente de determinação ajustado $\left(R^{2}\right)$ e valores de p dos modelos obtidos entre as medidas oculares em milímetros da câmara anterior (D1), tamanho do cristalino (D2), profundidade da câmara vítrea (D3) e diâmetro axial do olho (D4) e as variáveis morfométricas

(VM), diâmetro fronto-occipital (DFO) e diâmetro bizigomático (DBZ) do grupo de animais braquicefálicos da raça persa (GB). *Intervalo de confiança de $95 \%$. $(\mathrm{P}<0,05)$

\begin{tabular}{lcccc}
\hline Medida & VM & Parâmetro* & $\mathrm{R}^{2}$ & $\mathrm{P}$ \\
\hline D1 & Constante & 1,122 & 0,348 & $0,009^{* *}$ \\
& peso & 0,478 & & $<0,001^{* *}$ \\
D2 & idade & 0,073 & & $0,008^{* *}$ \\
& Constante & 8,469 & 0,108 & $<0,001^{* *}$ \\
D4 & idade & $-0,078$ & & $0,002^{* *}$ \\
(Fêmeas) & Constante & 18,215 & 0,119 & $<0,001^{* *}$ \\
D4 & peso & 0,371 & & $0,030^{* *}$ \\
(Machos) & Constante & 10,875 & 0,695 & $<0,001^{* *}$ \\
& DBZ & 1,120 & & $<0,001^{* *}$
\end{tabular}

Quadro 4. Equações obtidas para a estimativa das medidas oculares da câmara anterior (D1), tamanho do cristalino (D2), diâmetro axial do olho (D4), segundo o peso corporal (kg), idade (anos) e o diâmetro bizigomático $(\mathrm{cm})$ para gatos braquicefálicos (GB)

\footnotetext{
D1 $=1,122+0,478 \times \mathrm{kg}$ de peso $-0,073 \times$ idade

D2 $=8,469-0,078 \times$ idade

D4 fêmeas $=18,215+0,371 \times \mathrm{kg}$ de peso

D4 machos $=10,875+1,120 \times$ cm de diâmetro bizigomático
}

medidas da câmara vítrea (D3) não foram influenciadas significativamente por nenhuma das variáveis investigadas $(\mathrm{F}=0,574 ; \mathrm{p}=0,683)$. Os modelos resultantes podem ser vistos no Quadro 3, e as equações resultantes obtidas para GB no Quadro 4.

Para os gatos não braquicefálicos, as medidas oculares referentes à D1 puderam ser previstas significativamente pelo diâmetro fronto-occipital. Em referência as medidas da câmara vítrea (D3) e do diâmetro axial do bulbo ocular (D4), apresentaram relações significativas com o diâmetro bizigomático dos felinos. As medidas referentes à espessura do cristalino (D2) não foram influenciadas significativamente por nenhuma das variáveis que influenciaram esta medida nos braquicefálicos, as medidas oculares referentes à câmara anterior (D1) do bulbo ocular, não puderam ser previstas significativamente por nenhuma das variáveis investigadas $(p=0,350)$. Os modelos resultantes podem ser vistos no Quadro 5, e as equações resultantes obtidas para GNB no Quadro 6.

Quadro 2. Médias (intervalo de confiança 95\%) das medidas em milímetros da câmara anterior (D1), tamanho do cristalino (D2), profundidade da câmara vítrea (D3) e diâmetro axial do olho (D4), resultados das análises de covariância entre os gêneros (machos e fêmeas), controlando para idade (anos), peso (kg), diâmetros (cm) fronto-occipital (DFO) e diâmetro bizigomático

(DBZ) dos gatos, separados por raça (persa-braquicefálico - GB; sem raça definida - não braquicefálico GNB) $(\mathrm{P}<0,05)$

\begin{tabular}{ccccccc}
\hline & \multicolumn{5}{c}{ GB } & \multicolumn{3}{c}{ GNB } & Macho & P \\
\cline { 2 - 7 } & Fêmea & Macho & P & Fêmea & $3,67(3,49-3,86)$ & 0,717 \\
D1 & $3,18(3,01-3,36)$ & $2,81(2,48-3,36)$ & 0,081 & $3,62(3,44-3,81)$ & $3,67(3,49-7,87)$ & 0,657 \\
D2 & $7,97(7,75-8,19)$ & $8,11(7,69-8,53)$ & 0,602 & $7,69(7,40-7,96)$ & $7,59(7,31-7,87)$ & 0,605 \\
D3 & $8,06(7,81-8,31)$ & $7,56(7,01-8,04)$ & 0,107 & $7,83(7,56-8,11)$ & $7,73(7,45-8,00)$ & 0,605 \\
D4 & $19,56(19,38-19,74)$ & $18,64(18,30-18,99)$ & $<0,001^{*}$ & $19,29(18,94-19,63)$ & $19,14(18,80-19,49)$ & 0,576
\end{tabular}


Quadro 5. Parâmetros, coeficiente de determinação ajustado $\left(R^{2}\right)$ e valores de $p$ dos modelos obtidos entre as medidas oculares em milímetros da câmara anterior (D1), tamanho do cristalino (D2), profundidade da câmara vítrea (D3) e diâmetro axial do olho (D4) e as variáveis morfométricas

(VM), diâmetro fronto-occipital (DFO) e diâmetro bizigomático (DBZ) do grupo de animais sem raça definida (GNB). *Intervalo de confiança de $95 \%$. $(\mathrm{P}<0,05)$

\begin{tabular}{lcccc}
\hline Medida & Variável & Parâmetro & $\mathrm{R}^{2}$ & $\mathrm{P}$ \\
\hline D1 & Constante & 1,070 & 0,16 & 0,217 \\
& DFO & 0,343 & & $0,004^{* *}$ \\
D3 & Constante & 4,903 & 0,085 & $<0,001^{* *}$ \\
& DBZ & 0,422 & & $0,031^{* *}$ \\
D4 & Constante & 12,675 & 0,264 & $<0,001^{* *}$ \\
& DBZ & 0,960 & & $<0,001^{* *}$
\end{tabular}

Quadro 6. Equações obtidas para a estimativa das medidas oculares da câmara anterior (D1), profundidade da câmara vítrea (D3) e diâmetro axial do olho (D4), segundos os diâmetros fronto-occipital $(\mathrm{cm})$ e bizigomático $(\mathrm{cm})$ para gatos não braquicefálicos (GNB)

$\mathrm{D} 1=1,07+0,343 \times \mathrm{cm}$ de diâmetro fronto-occipital

D3 $=4,903+0,422 \times \mathrm{cm}$ de diâmetro bizigomático

D4 = 12,675 + 0,960 x cm de diâmetro bizigomático

\section{DISCUSSÃO}

Em gatos, são parcos os estudos referentes à biometria ultrassonográfica ocular que contemplem diferentes raças e suas relações com os parâmetros morfométricos. Dentre as raças felinas de importância comercial, a raça persa merece destaque em razão de sua alta comercialização no Brasil, sendo um dos fatores de escolha para sua inclusão neste estudo. Ademais, seu crânio largo e curto representa o padrão anatômico braquicefálico (Dyce, Sack \& Wesing 1997). Por outro lado, o grande número de gatos sem uma raça definida e com conformações craniais distintas, também motivou sua inclusão. A inclusão de gatos acima de um ano de idade (adultos), fundamentou-se no fato de não haver crescimento ósseo do crânio e nem do bulbo do olho após essa idade (Dyce, Sack \& Wesing 1997), de modo que a idade não fosse uma variável de comparação.

Parte dos trabalhos disponíveis sobre ultrassonografia ocular em felinos domésticos (Nathan et al. 1984, Moodie et al. 2001, Gonçalves et al. 2009), referem o uso de fármacos para a sedação ou anestesia dos animais submetidos ao exame. Neste estudo, foi possível a condução do exame nos 40 animais somente com a contenção manual e a dessensibilização corneana com colírio de proximetacaína corroborando o relatado por Gilger et al. (1998) que realizaram a ultrassonografia ocular em 15 gatos valendo-se de contenção manual e dessensibilização da superfície corneana com colírio de proparacaína a $0,5 \%$.

A utilização de transdutor microlinear nos felinos demonstrou-se viável, promovendo adequado contato com a superfície ocular, minimizando a formação de interfaces acústicas. 0 transdutor de $9 \mathrm{MHz}$, adotado neste estudo, conferiu imagens de alta qualidade e confiabilidade, visto que apresenta menor poder de penetração tecidual (Carvalho 2004). Gonçalves et al. (2009) também relataram a obtenção de valores fidedignos das medidas oculares de gatos sem raça definida, anestesiados, com o emprego de transdutor microconvexo de 7,5 MHz. Entretanto, com o transdutor microlinear de $9 \mathrm{MHz}$, não foi possível mensurar a espessura corneana, como relatado por Beserra et al. (2009), num estudo em cães, utilizando transdutor microconvexo e multifrequencial de 5 a $8 \mathrm{MHz}$. Aubin et al. (2003) utilizaram transdutor de alta frequência ( $50 \mathrm{MHz})$ em gatos e referiram ser possível mensurar a espessura da córnea, a profundidade da câmara anterior (D1) e da primeira porção do processo ciliar à base da íris e o ângulo iridocorneano. Bentley et al. (2003) relataram que transdutores de frequência superior a $20 \mathrm{MHz}$ possibilitaram a visualização de imagens de estruturas do segmento anterior do olho (córnea, cápsula posterior do cristalino e íris) devido a sua maior definição de imagem e poder de penetração, aproximadamente, 20 a $80 \mu \mathrm{m}$ e limite de penetração de 5 a 10 mm em gatos e cães.

Como fator favorável às mensurações da biometria ocular, ressalta-se o fato das mesmas terem sido obtidas pelo mesmo aparelho, além de serem realizadas por um examinador único e com rotina de exame, em consonância ao referido por Findl et al. (2003).

Os valores da biometria (D1, D2, D3 e D4) adquiridos neste estudo foram feitos pela mensuração direta das estruturas intraoculares, ao contrário do efetuado em outros estudos (Shufelt et al. 2005, Squarzoni 2011) em que o valor de D3 foi obtido pela diferença entre D1 e D4, sendo portanto, os valores encontrados fidedignos e validam os valores de D3, obtidos pela subtração de D1 e D2 de D4.

De acordo com o relatado por outros autores (Gilger et al. 1998, Gonçalves et al. 2000, Tuntivanich et al. 2007, Beserra et al. 2009, Gonçalves et al. 2009) a comparação entre os olhos direito e esquerdo, em ambos os grupos deste estudo, não diferiram à estatística. Porém, contrariando o relatado por estes autores, foi observada diferença significativa em D4 de machos e fêmeas no grupo GB, apresentando as fêmeas, medida superior a dos machos. Não foram encontrados em artigos publicados argumentos que justifiquem tal observação em detrimento ser este o primeiro estudo na raça persa, não permitindo contudo, estabelecer comparações com a literatura.

A observação de maiores medidas de D1 (câmara anterior) e D2 (cristalino), nos gatos não braquicefálicos e braquicefálicos, respectivamente, não puderam ser correlacionadas com outros dados da literatura capazes de elucidar as diferenças verificadas neste trabalho, sugerindo que maiores estudos devam ser conduzidos em gatos.

As medidas oculares dos gatos do grupo GNB encontram-se próximas as de outros estudos em gatos sem raça definida (Nathan et al. 1984, Gilger et al. 1998, Gonçalves et al. 2009, Konrade et al. 2012).

Baraldi et al. (2012), em estudo com gatos sem raça definida, verificaram que os valores biométricos oculares empregando o modo-B, comparativamente aos valores obtidos com o modo-A, apresentaram diferenças evidentes com relação a D4, contrariando o observado neste estudo em que as medidas D1, D2, D3 e D4, foram inicialmente obtidas em modo-B e, secundariamente, aplicando-se o modo-A não divergiram. 
Não há correlações entre as medidas oculares e os parâmetros morfométricos do crânio e corporais de gatos, assim, as equações lineares obtidas para as medidas de D1, D2, D3 e D4 em ambos os grupos não foram passíveis de comparação com a espécie. Beserra et al. (2009) realizaram estudo semelhante em cães sem raça definida e não braquicefálicos, e verificaram que os valores de D1, D3 e D4 foram influenciados significativamente pelo diâmetro fronto-occipital à exceção de D2. Neste estudo, nos animais do grupo não braquicefálico, as equações lineares demonstraram que D1 é influenciado significativamente pelo comprimento do crânio, de modo que quanto mais longo o crânio dos felinos maior a profundidade da câmara anterior. Ainda, observou-se influências significativas das medidas cranianas fronto-occipital sobre D3 e D4, coincidindo com o descrito por Beserra et al. (2009), levando a crer que quanto maior a largura do crânio destes gatos maior os valores de D3 e D4.

Para os gatos braquicefálicos, as equações lineares demonstraram haver influências das variáveis referentes à idade, ao peso e ao diâmetro bizigomático, uma vez que as medidas de D1 e D2 foram influenciadas pela idade, mesmo com todos os gatos sendo adultos. Ainda, D1 também foi influenciada pelo peso assim como D4 em fêmeas.Quanto à medida de $\mathrm{D} 4$ em machos, foi observado que a influência foi positiva para o diâmetro bizigomático. Pode-se sugerir também que as medidas da câmara anterior como as do cristalino são influenciadas pela idade, ressaltando que a câmara anterior também foi influenciada pelo peso, assim como o encontrado para o diâmetro axial do olho em fêmeas, o oposto do observado nos machos, nos quais o diâmetro axial do olho foi influenciado pelo diâmetro bizigomático. Em função da escassez de dados acerca de gatos braquicefálicos, observa-se a necessidade da realização de novos estudos biométricos como de curva de crescimento, a exemplo do estudo realizado em crianças (Fledelius et al. 1996), no qual o conhecimento das medidas oculares durante o desenvolvimento ocular, auxiliaram na identificação de anormalidades oculares ao nascimento, como em casos de microftalmia, e ao longo do seu desenvolvimento, ressaltando-se em casos de glaucoma.

Ademais, o conhecimento de tais medidas biométricas oculares pode em conjunto com outros exames, como a retinoscopia, favorecer a identificação de erros refracionais nos gatos braquicefálicos, como o que já fora realizado em gatos sem raça definida (Nathan et al. 1984, Moodie et al. 2001, Konrade et al. 2012), verificando que filhotes apresentam erro refracional maior quando comparados a gatos adultos (Konrade et al. 2012).

\section{CONCLUSÕES}

Considerando-se o grupo como um todo e a metodologia empregada, permite concluir que a câmara anterior dos gatos não braquicefálicos é maior que a dos braquicefálicos, ocorrendo o inverso em relação ao cristalino.

As fêmeas do grupo braquicefálico apresentaram diâmetro axial do bulbo do olho maior que os machos. Nesse grupo, verificou-se que o peso e a idade influenciaram o valor da câmara anterior, e o tamanho do cristalino sofre influência da idade.
O diâmetro axial do bulbo do olho nos machos demonstrou influência do diâmetro bizigomático e, nas fêmeas, do peso.

Nos não braquicefálicos, o diâmetro fronto-occipital influencia o valor da câmara anterior, enquanto a profundidade da câmara vítrea e o diâmetro axial do bulbo foram influenciados pelo diâmetro bizigomático.

\section{REFERÊNCIAS}

Adan C.B.D., Blay D., Yu M.C.Z., Freitas D. \& Allemann N. 2001. Ultra-sonografia ocular em suspeita clínica de endoftalmite. Arq. Bras. Med. Vet. Zootec. 64:423-428.

ARVO 2012. Statement for the use of animals. In Ophthalmic and Visual Research. Disponível em <http://www.arvo.org/About_ARVO/Policies/ Statement_for_the_Use_of_Animals_in_Ophthalmic_and_Visual_Research/> Acesso em 30 abr. 2013.

Atta H.R. 1999. New applications. In ultrasound technology. Brit. J. Ophthalmol. 12(83):1246-1249.

Aubin M.L., Powell C.C., Gionfriddo J.R. \& Fails A.D. 2003. Ultrasound biomicroscopy of the feline anterior segment. Vet. Ophthalmol. 6:15-17.

Baraldi T.G., Mamprim M.J., Brandão C.V.S., Sartor R., Perches C.S. \& Ackermann C.L. 2012. Biometria ultrassonográfica modo-A e modo-B do globo ocular de gatos domésticos. Vet. Zootec. 19:20-23.

Bentley E., Miller P.E. \& Diehl K.A. 2003. Use of high-resolution ultrasound as a diagnostic tool in veterinary ophthalmology. J. Am. Vet. Med. Assoc. 223:161-122.

Beserra P.S., Sales G.A., Santana E.J.M., Miranda S.A., Brito A.B., Nickolak E. \& Domingues S.F.S. 2009. Relação entre a biometria ultra-sonográfica em modo B do bulbo ocular e os diâmetros fronto occipital e bizigomático em Canis familiaris. Pesq. Vet. Bras. 29:286-290.

Bhatt A.B. Schefler A.C., Feuer W.J., Yoo S.H. \& Murray T.G. 2008. Comparison of predictions made by the intraocular lens masters and ultrasound biometry. Arch. Ophthalmol. 126:929-933.

Carvalho C.F. 2004. Bases físicas da formação da imagem ultra-sonográfica, p.1-7. In: Carvalho C.F. (Ed.), Ultra-sonografia em Pequenos Animais. Roca, São Paulo.

Corrêa Z.M.S., Goldhardt R., Marcon A.S. \& Marcon I.M. 2002. Achados ecográficos em pacientes com catarata total. Arq. Bras. Oftalmol. 65:609-613.

Dyce K.M., Sack W.O. \& Wesing C.J.G. 1997. Tratado de Anatomia Veterinária. Guanabara Koogan, Rio de Janeiro, p.258-276.

Eisemberg H.M. 1985. Ultrasonography of the eye and orbit. Vet. Clin. North Am., Small Anim. Pract. 15:1263-1274.

Findl O., Kriechbaum K., Sacu S., Kiss B., Polak Neep J., Schild G., Rainer G., Maca S., Petternel V., Lackner B. \& Drexler W. 2003. Influence of operator experience on the performance of ultrasound biometry compared to optical biometry before cataract surgery. J. Cataract Refract. Surg. 29:1950-1955.

Fledelius H.C. \& Christensen A.C. 1996. Reappraisal of the human ocular growth curve in fetal life, infancy, and early childhood. Brit. J. Ophthalmol. 80:918-921.

Gilger B.C., Davidson M.G. \& Howard P.B. 1998. Keratometry, ultrasonic, and prediction of intraocular lens power in the feline eye. Am. J. Vet. Res. 59:131-134.

Gomes D., Otsuki D.A., Lisak R. \& Safatle A.M.V. 2013. Atrofia progressiva de retina em cães da raça cocker spaniel. Ciência Rural 43:1405-1414.

Gonçalves G.F., Leme M.C., Romaggnoli P., Eurides D. \& Pippi N.L. 2009. Biometria ultra-sonográfica bidimensional em tempo real de bulbo ocular de gatos domésticos. Ciênc. Anim. Bras. 10:829-834.

Gonçalves G.F., Pippi N.L., Raiser A.G., Mazzanti A., Oliveira S.T., Neves J.P., Leotte A.M. \& Hintz C.W. 2000. Biometria ultra-sonográfica bidimensional em tempo real do globo ocular de cães. Ciência Rural 30:417-420.

Hernandez-Guerra A.M., Rodilla V. \& López-Murcia M.M. 2007. Ocular biometry in the adult anesthetized ferret (Mustela putorius furo). Vet. Ophthalmol. 10:50-52. 
Hijar M.V. 2008. Ultra-sonografia ocular, p.49-62. In: Herrera D. (Ed.), Oftalmologia Clínica em Animais de Companhia. MedVet, São Paulo.

Junior E.F.R. 2008. Ultra-sonografia pré-cirúrgica da lente do segmento posterior de cães portadores de catarata. Dissertação Mestrado em Cirurgia Veterinária, Faculdade de Ciências Agrárias e Veterinárias, Universidade Estadual Paulista, Jaboticabal. 53p.

Konrade K.A., Hoffman A.L., Ramey K.L., Goldenberg R.B. \& Lehenbauer T.W. 2012. Refractive states of eyes and associations between ametropia and age, breed, and axial globe length in domestic cats. Am. J. Vet. Res. 73:279-284.

Kubal W.S. 2008. Imaging of orbital trauma. Radiographics 28:17291739.

Lehmkuhl R.C., Almeida M.F., Mamprim M.J. \& Vulcano L.C. 2010. B-mode ultrasonography biometry of the Amazon Parrot (Amazona aestiva) eye. Vet. Ophthalmol. 13:26-28.

Lima L., Montiani-Ferreira F., Tramontin M., Santos L.L., Machado M., Lange R.R. \& Russ H.H.A. 2010. The chinchilla eye: morphologic observations, ecobiometric findings and reference values for selected ophthalmic diagnostic test. Vet. Ophthalmol. 13:14-25.

Lucena D.R., Ribeiro J.A.S., Folgosa M.S.P.A., Lucena L.R. \& Jorge R. 2009. Ultrasonography-guided scleral buckle retinopexy and cryotherapy: case report. Arq. Bras. Oftalmol. 72:243-246.

Lupinacci A.P.C., Vanini R., Isaac D.L.C., Ghanem V.C. \& Arieta C.E.L. 2004. Importância da ultra-sonografia ocular na avaliação pré-operatória de pacientes com catarata total. Arq. Bras. Oftalmol. 67:33-36.

Luyet C., Eichenberger U., Moriggl B., Remonda L. \& Greif R. 2008. Real-time visualization of ultrasound-guided retrobulbar blockade: an imaging study. Brit. J. Anesth. 101:855-859.

Maia F.B.N., Pigatto J.A.T., Guedes P.M. \& Barros P.S.M. 2003. Ocular biomicroscopy (UBM) in Yacare Caiman (Caiman Yacare). Vet. Ophthalmol. 6:351-366.

Martins B.C., Lima F.S. \& Laus J.L. 2010. Simultaneous mode A and mode B ecobiometry of senile cataractous eyes in dogs. Arq. Bras. Med. Vet. Zootec. 62:24-46.

Miglior S., Albe E., Guareshi M., Mandelli G., Gomarasca S. \& Orzalesi N. 2004. Intraobserver and interobserver reproducibility in the evaluation of ultrasonic pachymetry measurements of central corneal thickness. Brit. J. Ophthalmol. 88:74-177.

Moodie K.L., Hashizume N., Houston D.L., Hoopes P.J., Demidenko E., Trembly B.S. \& Davidson M.G. 2001. Postnatal development of corneal curvature and thickness in the cat. Vet. Ophthalmol. 4:267-272.

Murphy C.J., Zadnick K. \& Mannis M.J. 1992. Myopia and refractive error in dogs. Invest. Ophthalmol. Vis. Sci. 33:2459-2463.
Mutti D.O., Zadnik K. \& Murphy C.J. 1999. Naturally occurring vitreous chamber-based myopia in the Labrador Retriver. Invest. Ophthalmol. Vis. Sci. 40:1577-1584.

Nathan J., Crewther S.G., Crewther D.P. \& Kiely P.M. 1984. Effects of retinal image degradation on ocular growth in cats. Invest. Ophthalmol. Vis. Sci. 25:1300-1306.

Nunnery C.M., Barrie K.P., Wiendner E.B., Gellatt-Nicholson K.J. Plummer C.E. \& Brooks D.E. 2008. Ocular ultrasound findings in the Asian elephant Elephas maximus. Vet. Ophthalmol. 11:412-419.

Ribeiro A.P., Santos N.L., Silva V.C., Campos A.F., Teixeira I.A.M.A. \& Laus J.L. 2010. Ultrassonografhic and ecobiometric findings in the eye of adult goats. Ciência Rural 40:568-573.

Ribeiro A.P., Miguel L.S., Juliana P.R., Teixeira I.A.M.A., Souza A.L.G. \& Laus J.L. 2009. Ocular biometry in a colony of Saanen goats with different ages. Vet. Ophthalmol. 12:313-317.

Schiffer S.P., Rantanen N.W., Leary C.A. \& Bryan G.M. 1982. Biometric study of the canine eye, using A-mode ultrasonography. Am. J. Vet. Res. 43:826-830.

Shufelt C., Fraser-Bel S., Ying-Lai M., Torres M. \& Varmar R. 2005. The Los Angeles Latino Eye Study Group: refractive error ocular biometry and lens opalescence in an adult population. Los Angeles Latino Eye Studt. Invest. Ophthalmol. Vis. Sci. 46:4450-4460.

Silva M.L., Martins B.C., Ribeiro A.P., Souza A.L. \& Laus J.L. 2010. A and B modes echobiometry in cataractous and noncataractous eyes of English Cocker Spaniel dogs. Arq. Bras. Med. Vet. Zootec. 62:1080-1085.

Soares A.M.B., Laus J.L., Siqueira Y.H. \& Marsillac P. 1998. Ultra-sonografia bidimensional em tempo real do bulbo ocular de cães (Canis familiaris Linnaeus, 1758) com opacificação de meios transparentes. Emprego do transdutor mecânico setorial de 7,5 MHz com almofada de recuo. Ciência Rural 28:591-599.

Squarzoni R. 2011. Biometria ocular e sua relação com sexo, idade, tamanho e peso em cães da raça Cavalier King Charles Spaniel. Tese de Doutorado em Clínica Cirúrgica Veterinária, Faculdade de Medicina Veterinária e Zootecnica, Universidade de São Paulo, São Paulo, SP. 95p.

Tartarella M.B., Takahagi R.U., Braga A.P. \& Filho J.B.F. 2013. Persistent fetal vasculature: ocular features, management of cataract and outcomes. Arq. Bras. Oftalmol. 76:185-186.

Tuntivanich N., Petersen-Jones S.M., Steibel J.P., Jhonson C. \& Forcier J.Q. 2007. Postnatal development of canine axial globe length measured by B-scan ultrasonography. Vet. Ophthalmol. 10:2-5.

Williams D.L. 2004. Lens morphometry determined by B-mode ultrasonography of the normal and cataractous canine lens. Vet. Ophthalmol. 7:91-95. 